\title{
IRC in ADPKD: dati consolidati e quesiti ancora aperti. Update diagnostico-terapeutico e Qol
}

\author{
Fedele Lasaponara ${ }^{1,2}$ \\ ${ }^{1}$ ASL TO3, Torino \\ ${ }^{2}$ Clinica Sedes Sapientiae, Torino
}

\begin{abstract}
ADPKD and ESRD: established data and still open questions. A diagnostic-therapeutic and Qol upgrade
Experiences got from the most recent Literature are reported regarding early diagnosis techniques and targeted and personalized therapies. Main care is focused on the quality of life and psychological support at every stage of the disease. We investigated all the issues regarding women, considering pregnancy as well. The key role of the family support in the management of this chronic and disabling disease is highlighted, beside the crucial help given by the Patient Associations. Peritoneal dialysis is an effective alternative therapy to hemodialysis at the end stage renal failure, even if renal transplantation (single or combined with liver) is still considered the gold standard. It is the optimal solution regarding time and quality of life, management of complications and social cost of replacement therapy. We reported the state of the art about pre-transplantation nephrectomy. A wide debate about indications and timing of surgery still exists. Even if less invasive techniques are now available and spread (pure laparoscopy, hand assisted laparoscopy, minimally invasive open nephrectomy), the most recent data don't support the routine nephrectomy. Surgery should be considered only in case of specific complications and in order to get enough space to perform a safe and low-risk transplantation.
\end{abstract}

Keywords: Diagnosis, Dialysis, Kidney transplantation, Medical therapy, Polycystic kidney disease, Quality of life

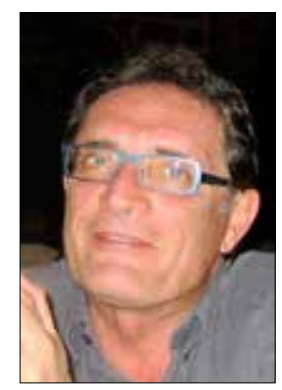

Fedele Lasaponara

\section{Introduzione}

La malattia renale policistica autosomica dominante ( $A D P K D)$ è nota da più di 300 anni ed è stata considerata per molto tempo una malattia rara e incurabile; oggi, invece, è diagnosticata più frequentemente e varie sono le strategie che ne hanno migliorato durata e qualità di vita (Qol).

Epidemiologia ed Eziologia. Diverse alterazioni patologiche su base ereditaria possono portare allo sviluppo di cisti: I'ADPKD interessa, a seconda delle statistiche, da 1:400 a 1:1000 soggetti nella popolazione generale; è la patologia renale ereditaria più

Accepted: January 20, 2016

Published online: February 19, 2016

Indirizzo per la corrispondenza:

Dr. Federico Lasaponara

Corso Massimo d'Azeglio 108

10126 Torino

fedele45@hotmail.com frequente: più di 12 milioni di casi nel mondo ( 600.000 solo negli USA), dove ogni anno vengono diagnosticati 5-6000 nuovi casi, il $45 \%$ dei quali nella quinta decade di età $(1,2)$. Complessivamente, è causa del $5 \%$ di tutte le insufficienze renali terminali (ESRD) e rappresenta un grave onere di assistenza sanitaria nei paesi sviluppati (3).

La mutazione genetica può interessare tre diverse localizzazioni: nell' $85-90 \%$ dei casi si tratta della mutazione del gene PKD1 (cromosoma 16), braccio corto (16p 13.3), nel 10-15\% del gene PKD2 (cromosoma 4), braccio lungo (4q 21) e, nel 5\%, del gene PKD3.

Anatomia patologica. Macroscopicamente, i reni, aumentati di dimensione e peso, appaiono di superficie irregolare; all'osservazione microscopica della parete cistica è presente un epitelio cubico, mentre il parenchima circostante si presenta con pochi glomeruli, con ialinosi e fibrosi.

In genere, solo poche cisti renali vengono rilevate prima del 30 anni, ma, a partire dalla quinta decade di vita, si trovano, nella maggior parte dei pazienti, migliaia di cisti renali (Fig. 1). A malattia stabilizzata, i reni possono raggiungere fino a $40 \mathrm{~cm}$ di lunghezza e pesare fino a $8 \mathrm{~kg}$ (4). L'ingrandimento del rene è correlato a complicanze come dolore, ematuria, ipertensione e insufficienza renale. Oltre che a livello renale, le cisti si possono sviluppare nel fegato (circa nell' $80 \%$ dei pazienti), nel pancreas $(10 \%)$, nella milza, nell'aracnoide e nelle vescicole seminali. 


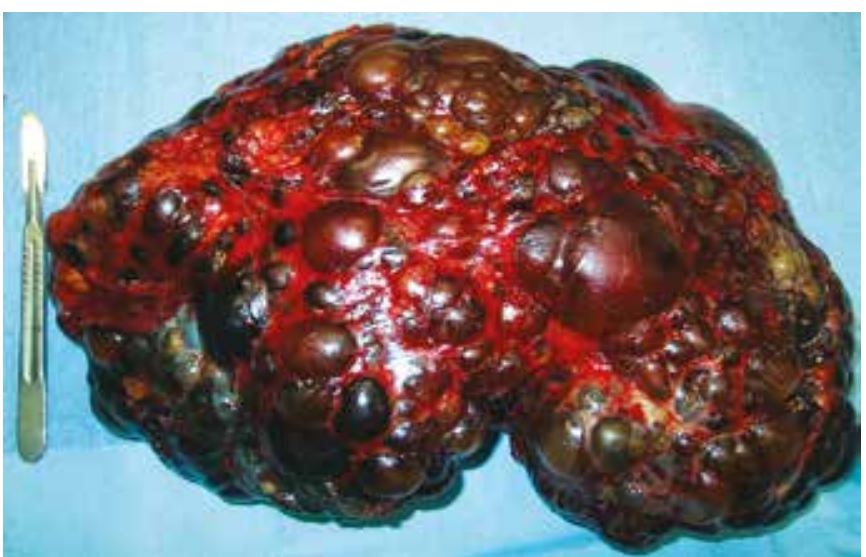

Fig. 1 - Rene policistico.

Tra le lesioni non cistiche, possono essere presenti aneurismi intracranici, anomalie delle valvole cardiache (in circa $1 / 3$ dei pazienti: più spesso prolasso della mitrale, ma anche alterazioni della valvola aortica), ernie (ombelicali, iatali, inguinali) e diverticoli del colon.

Sintomatologia. In genere, i soggetti ADPKD di età tra i 20 e i 30 anni sono asintomatici: sovente i sintomi esordiscono nella III e nella IV decade e dalla $V$ può instaurarsi un'insufficienza renale cronica (IRC); la forma PKD2 ha un esordio più tardivo e una minore progressione del danno renale rispetto alla forma PKD1.

L'ipertensione arteriosa lieve o moderata compare precocemente; ma, tra i sintomi principali, possono presentarsi anche ematuria, infezioni delle vie urinarie (UTI) e dolore lombare gravativo. Lo sviluppo numerico e volumetrico delle cisti renali ed epatiche è spesso motivo di ingombro addominale, con alterazioni gastro-intestinali e nutrizionali anche gravi e dolore, per la pressione che si crea sulla parete addominale, in regione lombare e al dorso, ma anche per la compressione degli organi contigui. La forma acuta può essere dovuta a emorragia intracistica (con distensione capsulare), rottura cistica o infezione, così come alla frequente litiasi urinaria (specialmente calcoli di urati o di ossalato di calcio) (5). È definito cronico il dolore di durata superiore alle 4-6 settimane consecutive, causato dal volume e dal peso degli organi (localizzazione muscolo-scheletrica o lombare) oppure legato in modo diretto alla distensione della capsula renale e/o epatica o alla compressione degli organi contigui. Tra le manifestazioni algiche è importante ricordare anche il dolore toracico, i dolori radicolari irradiati alle anche e agli arti inferiori e la cefalea cronica (presente con percentuali sino al 48.5\%). II dolore è un sintomo comune, ma la sua presentazione, la sua gravità, la sua frequenza e la sua posizione spesso non sono dettagliatamente caratterizzate in Letteratura, anche perché non sempre viene segnalato e correlato con I'ADPKD. Frequentemente, i pazienti fanno ricorso all'auto-medicazione: ai Sanitari il compito e l'impegno di dare informazioni circa I'uso di farmaci antalgici che possono influire negativamente sulla funzione renale; il trattamento sintomatico deve escludere il ricorso ai FANS, ma basarsi, invece, sul paracetamolo, al quale far seguire, se necessario, blandi oppioidi (Tramadolo) e, quindi, un terzo livello di terapia con oppioidi maggiori (Morfina). Possono essere necessari anche interventi invasivi quali la denervazione renale, effettuata anche per via laparoscopica (ma che può essere causa di importanti complicanze ipotensive), o la denervazione percutanea transcatetere con coagulazione dei tessuti periarteriosi a livello renale. II dolore a livello epatico o di altri organi può essere trattato con blocco selettivo del plesso celiaco o del nervo splancnico, utilizzando agenti neurolitici o radiofrequenze.

È necessario che il medico comprenda la cronicità della sindrome dolorosa, che può essere invalidante e alterare la qualità della vita, e che vigili affinché non si crei una potenziale cascata di trattamenti, molti dei quali anche non propriamente farmacologici (6).

\section{Diagnosi}

In genere, la diagnosi di ADPKD clinicamente manifesta è semplice: il paziente si presenta con i reni ingranditi, cisti renali multiple bilaterali e storia familiare positiva. Per lo screening pre-sintomatico dei soggetti a rischio e per la valutazione dei potenziali donatori di rene in famiglie con ADPKD, è ampiamente utilizzata l'ecografia renale: prendendo come riferimento i risultati dell'analisi del DNA come il "gold standard" per la diagnosi di malattia, i criteri diagnostici ecografici età-dipendente hanno offerto elevati valori predittivi positivi e negativi (7) (Tab. I).

II valore predittivo positivo (VPP) di questi criteri è del $100 \%$ in tutte le fasce d'età menzionate e il valore predittivo negativo (VPN) è dell'85.5\% per la fascia 15-29 anni, del 95\% per le due fasce $30-39$ e $40-59$ anni e del $100 \%$ oltre i 60 anni (4). L'ecografia, esame sicuro e di basso costo, è, quindi, la tecnica utilizzata per il primo livello di diagnosi: rivela con sicurezza le cisti superiori o uguali a $1 \mathrm{~cm}$ di diametro ed è altamente sensibile per la diagnosi di malattia nell'adulto; riguardo al monitoraggio periodico dell'ADPKD, vi sono, invece, in Letteratura, maggiori perplessità.

Posta la diagnosi, la tomografia assiale computerizzata (TC) e la risonanza magnetica nucleare (MRI) in 3D rappresentano gli esami più sensibili nel visualizzare cisti di piccole dimensioni e nel quantificare volumetricamente la malattia renale, permettendone un più accurato monitoraggio (8). Entrambe le metodiche, però, non sono scevre da rischi: la TC

TABELLA I - "Criteri ecografici classici" utilizzati per porre diagnosi di malattia policistica renale

1) almeno 3 cisti (bilaterali o unilaterali) in soggetti tra i 15 e i 29 anni

2) almeno 2 cisti per rene nei soggetti tra i 30 e i 59 anni

3) almeno 4 cisti per rene nei soggetti oltre i 60 anni 
determina l'esposizione a elevate dosi di radiazioni e, spesso, al mezzo di contrasto iodato, mentre la MRI utilizza il gadolinio e dovrebbe essere evitata per il rischio di fibrosi nefrogenica sistemica (NSF) con filtrato glomerulare (GFR) $<30 \mathrm{~mL} /$ $\min (8)$. Nei pazienti con IRC, occorre valutare il rapporto rischio/beneficio dovuto alla MRI: la condizione ideale sarebbe poter evitare l'uso del gadolinio ottenendo ugualmente immagini precise ad alta risoluzione; sono stati fatti vari studi utilizzando misurazioni T1 o T2 pesate in assenza di gadolinio: in entrambi i casi, sono necessari maggiori tempi per l'elaborazione delle immagini e la valutazione della misurazione del volume renale è più difficoltosa. Nonostante questi limiti, è preferibile misurare la volumetria delle cisti senza il mezzo di contrasto. La MRI consente, oltre alla valutazione dei parametri anatomici (volume renale totale, volume totale delle cisti e percentuale di cisti sul volume totale), anche la valutazione di quelli emodinamici (flusso plasmatico renale e resistenze vascolari intrarenali) e permette, quindi, una valutazione funzionale, anche se indiretta.

Test genetici. La caratterizzazione dettagliata delle strutture genomiche PKD1 e PKD2 ha fornito tutti i reagenti essenziali necessari per eseguire test genetici (DNA linkage analysis e Gene-Based Mutation Screening) per la diagnosi molecolare (9). La diagnosi presintomatica di ADPKD in genere viene effettuata mediante ecografia renale, tuttavia, specialmente nei pazienti con risultati di imaging equivoci, la diagnosi molecolare ha un ruolo in soggetti con una storia familiare negativa e nei casi in cui individui giovani a rischio e con ecografia negativa vengono valutati quali potenziali donatori di rene per trapianto da vivente. Nel trapianto di rene da donatore vivente familiare (LRKD), l'interesse principale è escludere una diagnosi di ADPKD: gli studi di imaging non lo consentono, specialmente nei soggetti giovani, ed è indicata l'esecuzione del test genetico che può rendere sicura la valutazione e aumentare le possibili donazioni: la presenza di cisti e di un test genetico negativo implica, comunque, una valutazione multidisciplinare (9).

Il principale vantaggio della metodica DNA linkage analysis è che, se è disponibile una grande famiglia con diversi membri affetti, si può determinare se il soggetto è un vettore obbligato di malattia e non è indispensabile l'identificazione della mutazione (che può essere difficile, specialmente in PKD1); richiede informazioni cliniche e campioni di sangue di molti individui e dà risultati poco significativi in caso di famiglie non numerose. L'altro test (Gene-Based Mutation Screening) richiede un solo campione ematico del soggetto in esame, ma è costoso e poco sensibile: una mutazione si trova in non più di $2 / 3$ dei soggetti testati ed è utile solo quando è positivo.

Stadiazione funzionale. La malattia ha un andamento cronico e può condurre a IRC: nell'attuale pratica clinica, in tutte le patologie renali, la valutazione del GFR è considerata il gold standard per la quantificazione del tasso di progressione; la sua misurazione periodica consente di valutare variazioni di attività funzionale anche dalle prime fasi patologiche.
Tuttavia, in caso di ADPKD, il notevole numero di nefroni intatti può compensare la perdita del parenchima funzionante, rendendo meno attendibile l'esame; d'altra parte, le misurazioni sequenziali del volume renale possono quantificare il tasso di progressione della malattia prima di sensibili variazioni del GFR. Lo studio genotipico ha cambiato il modo di giudicare la prognosi della perdita funzionale renale: gli individui con mutazioni PKD2 sviluppano IRC circa 15 anni più tardi rispetto a quelli con mutazioni PKD1 (10). Tuttavia, all'ispezione clinica, un individuo con una mutazione PKD2 non presenta alterazioni differenti da un soggetto con una mutazione PKD1.

Dalgaard, già nel 1957, pubblicò uno studio che dimostrava una forte evidenza a sostegno del parere che le cisti renali fossero causa dell'IRC. L'Autore sottolineò, nel suo celebre e storico lavoro, che, in individui giovani, ma anche sino all'ottava decade, l'aumento delle dimensioni dei reni precedeva la perdita funzionale: reni di dimensioni palpabili e dolore sono presenti prima dell'esordio dell'uremia (11). Molti studi, precedenti e successivi a questo, hanno trovato un'associazione inversa tra dimensione del rene policistico e livello di GFR. In uno studio trasversale, Thomsen et al. (12) per primi utilizzarono l'imaging radiologico per determinare il volume renale (con funzione renale normale o alterata) e dimostrarono una chiara associazione tra volume renale totale e diminuzione della clearance. Oggi, studi trasversali e longitudinali nel soggetto ADPKD e in modelli animali di malattia cistica renale (PKD) hanno confermato che ingrandimento delle cisti e aumento delle dimensioni renali sono fattori importanti nello sviluppo di IRC a esordio tardivo. La crescita volumetrica delle cisti e la perdita nefronica conducono a una riduzione funzionale quando il rene raggiunge i $1500 \mathrm{~mL}$ e sono collegate a un calo progressivo del GFR del $5 \%$ per anno (circa $5 \mathrm{~mL}$ ) min/anno). La proteinuria $>300 \mathrm{mg} / \mathrm{dL}$ si presenta nel $25 \%$ dei pazienti in età adulta, ma raramente supera il valore di 1g/dL (13).

L'IRC è la temibile e temuta conseguenza di tutti i disturbi renali progressivi: la maggior parte delle condizioni che portano a IRC, come la glomerulonefrite, il diabete e l'ipertensione vascolare, hanno effetti primari o secondari sui glomeruli con alterazione del GFR, che viene considerato primo indicatore di progressione della malattia. La gravità di altre malattie renali croniche che non hanno origine a livello glomerulare (ADPKD, nefrite tubulo-interstiziale, malformazioni congenite e tubulopatie ereditarie) è, comunque, ugualmente valutata in base all'alterazione del GFR. Lo studio e lo sviluppo di trattamenti atti a rallentare la progressione patologica di malattie croniche non glomerulari, quindi, possono essere compromessi dall'utilizzo del solo GFR come end point primario di valutazione. D'altra parte, l'analisi del decorso della malattia è, altrimenti, difficile, dal momento che nessuna Organizzazione Sanitaria sarebbe evidentemente disponibile, al fine di determinare l'efficacia di un presidio terapeutico proposto, a sopportare i costi di uno studio clinico della durata di 20-40 anni. Le misurazioni del GFR possono essere particolarmente fuorvianti nel 
determinare la progressione di malattia nel caso dell'ADPKD: le cisti si sviluppano alla nascita e sono, senza dubbio, causa di grave morbilità nel lungo termine, molto prima che si instauri I'insufficienza renale; i reni, infatti, hanno notevoli capacità di compensazione della perdita di unità di filtrazione glomerulare. Il dosaggio urinario dell'Epidermal Growth Factor (uEGF) nei soggetti con una malattia renale cronica (CKD) consente la prognosi di progressione. La diminuzione della proteina EGF è un indice altamente sensibile della probabilità di peggioramento della CKD e un predittore indipendente del rischio di progressione. Rispetto ai parametri clinici standard l'uEGF migliora l'attività predittiva di eventi di malattia in varie popolazioni CKD ad ampio spettro eziopatogenetico e ai vari stadi patologici: l'individuazione tempestiva dei pazienti a più alto rischio di progressione può aumentare l'efficacia della tempistica terapeutica, preservando più a lungo la funzione renale e garantendo una migliore Qol (14).

\section{ADPKD e Qol}

La patologia policistica renale non solo determina importanti esigenze di cura, ma anche importanti aspetti psicologici e sociali: si è di fronte, sovente, a effetti psicofisici in grado di compromettere la Qol e il benessere del paziente e del suo contesto sociale e familiare. Un basso livello di Qol relativo alla salute (HQL) è associato all'evoluzione della CKD e alla mortalità allo stadio terminale della malattia. Pertanto, sono in corso studi sulle determinanti psicologiche della $\mathrm{HQL}$, che tentano di valutare se l'accettazione della malattia e l'impatto delle caratteristiche di personalità sull'accettazione contribuiscano a migliori condizioni di salute fisiche e mentali (PHQL e MHQL) (15). Nella cura di questi pazienti, è, quindi, importante anche la valutazione psicologica, al fine di offrire un approccio e interventi specifici tendenti a migliorare la Qol, con migliori risultati sulla salute del soggetto e un minor numero di ricoveri ospedalieri (16-19).

Un approccio olistico e la percezione del sostegno offerto dal Nefrologo possono contribuire alla scelta del trattamento più appropriato, con migliori compliance e risultati terapeutici (20).

QoL personale. In considerazione della natura ereditaria e cronica e delle importanti associazioni patologiche dell'ADPKD, soggetti portatori di policistosi renale possono lamentare valori di PCS (Physical Component Summary) e di MCS (Mental Component Summary) inferiori rispetto alla popolazione generale. Uno studio di coorte prospettico osservazionale, comprendente pazienti ADPKD e altre popolazioni, caratterizzato da un definito livello di funzione renale predialitico, ha tenuto conto dei fattori biochimici e genetici, delle variabili cliniche e delle necessità farmacologiche e dialitiche; si è osservato che, come in altre popolazioni CKD, il questionario SF-36 non fornisce la sensibilità necessaria per individuare un'insoddisfazione anche rilevante di Qol e risulta inadeguato nel rilevare i cambiamenti relativi al crescente onere della patologia cistica con l'età. Non sono, peraltro, disponibili, per I'ADPKD, PRO (patient-reported outcomes) validati.

È nota l'associazione tra le dimensioni renali, gli episodi di ematuria e il dolore, così come è nota la stretta relazione tra le dimensioni del rene e il GFR, ma, con il questionario SF-36, non è possibile evidenziare il rapporto esistente tra l'aumento del volume renale e PCS, MCS o punteggi dei singoli componenti (21). In soggetti ADPKD, le caratteristiche della forma renale, la disposizione delle cisti e la risposta infiammatoria sistemica possono contribuire in modo particolarmente significativo al dolore più del singolo dato volumetrico. Sono state prese in considerazione anche altre complicanze correlate: ematuria, infezioni e calcificazioni; nell'insieme, è evidente che i pazienti ADPKD, proprio per la bassa Qol, sono generalmente molto motivati a far fronte alla loro malattia. Non c'è, quindi, una stretta associazione tra dimensioni del rene e dolore: una possibile spiegazione è che il volume delle cisti e la loro localizzazione siano più importanti del volume totale dei reni, a meno che questi non superino i $1000 \mathrm{~mL}$. Anche nei confronti della perdita funzionale (calo del GFR) non c'è una correlazione con il dolore e ne consegue che il paziente riduce la propria attività non a causa del dolore. II senso di ripienezza addominale è, invece, correlato, specialmente nel sesso femminile, al calo del GFR: ciò è dovuto a un maggior ingrandimento della massa epatica, dovuta alla responsività delle cisti del fegato agli estrogeni; ciò comporta, nel sesso femminile, una maggiore interferenza con le normali attività quotidiane, specialmente in occasione del ciclo mestruale (22).

Si è visto che dolore e uso di farmaci antiipertensivi, età e indice di massa corporea non hanno una correlazione significativa con il punteggio PCS, mentre il livello di istruzione e il reddito sono positivamente correlati, come è stato riportato anche in altre CKD (sia pre-ESRD che ESRD). Al momento della diagnosi, spesso il dolore è il sintomo principale: è, quindi, indispensabile raccoglierne una precisa e dettagliata documentazione circa frequenza e gravità, per affrontare i problemi che questo sintomo ha sulla vita dei pazienti. I quadri algici sono generalmente più diffusi e più frequenti nel paziente con ADPKD di quanto non venga segnalato spontaneamente: non è sufficiente essere soltanto attenti a recepire la parola "dolore" e prescrivere un farmaco analgesico; il Sanitario deve, invece, essere vigile e percettivo e ricercare la precisa eziologia per proporne il trattamento più consono, spesso anche non farmacologico. È auspicabile, quindi, un'attenta comprensione della cronicità della sindrome dolorosa, che può essere fortemente invalidante e alterare gravemente la Qol, al fine di approntare consoni approcci terapeutici specifici. Devono essere indagate la sede del dolore, addominale, dorsale, dorsale con irradiazione alle cosce e alle gambe, toracico, cefalico, e l'entità, monitorizzata con appositi questionari, come l'"Headache and pain in polycystic kidney disease project" $(23,24)$.

Come riportato sopra, PCS e MCS, rilevati mediante questionario SF-36, in pre-dialisi, sono simili a quelli ottenuti in altre malattie croniche: questo dato porta a considerare 
come il questionario non abbia una sensibilità e una specificità adeguate a rilevare le variazioni di Qol che si verificano in pazienti con ADPKD: sono necessari questionari affidabili e validati, atti a valutare accuratamente lo stato fisico e mentale in questi pazienti (21). Ansia e depressione, dovute in particolare a una minore speranza di vita, sono frequenti nei pazienti CKD e interessano circa il 60\% dei soggetti ADPKD.

La Qol nei soggetti sottoposti a terapia sostitutiva renale, riguardo alla partecipazione ad attività della vita quotidiana (funzione fisica, viaggi, tempo libero e lavoro), è stata oggetto di numerosi studi. Purnell, nel 2013, ha pubblicato una review completa della Letteratura disponibile; da questo studio si rileva che i pazienti con trapianto di rene possono sperimentare migliori tassi di partecipazione rispetto ai pazienti sottoposti a dialisi, mentre non ci sono significative differenze tra i soggetti sottoposti a emodialisi e quelli sottoposti a dialisi peritoneale (25). È utile, nella presa in carico di questi pazienti, anche la valutazione psicologica (26), che consente approcci e interventi specifici per migliorare la Qol, con una più proficua gestione di malattia e riduzione del numero di ricoveri ospedalieri (27).

La patologia cistica renale non deve essere motivo di astensione da attività sportive, che, anzi, in molti casi sono raccomandabili; è opportuno evitare sport con contatto fisico per possibili traumi con rottura di cisti ed emorragie interne, come box, rugby, football americano e così via.

Gravidanza. Nel sesso femminile, contraccezione e possibilità di gravidanza rivestono un'importanza rilevante: in età fertile, quando non è programmata una gravidanza, la donna con ADPKD deve essere ben informata circa le reali possibilità contraccettive disponibili, tenendo presente che è preferibile evitare anticoncezionali orali a base di estrogeni, che, spesso, causano aumenti pressori e accrescimento delle cisti epatiche. La possibilità di intraprendere una gravidanza è reale e fattibile; rispetto alla popolazione generale, si ha, però, un incremento del $20-30 \%$ del rischio di aborto spontaneo e, trattandosi di una malattia a trasmissione genetica dominante, la coppia deve tenere presente il fatto che il 50\% dei figli sarà portatore dell'alterazione genetica. È fondamentale che la donna consideri attentamente quanto I'ADPKD potrebbe influire sulla gravidanza, ma anche quanto questa, a sua volta, possa deteriorare la funzione renale. Prima di programmare una gravidanza, qualora la paziente fosse in sovrappeso, è necessario un congruo calo ponderale; devono essere accuratamente monitorizzati tutti i problemi clinici che possono essere influenzati negativamente dalla gravidanza e devono essere sospese o sostituite le terapie farmacologiche che possono alterare lo sviluppo del nascituro (farmaci ipotensivi di comune utilizzo in ADPKD come ACE inibitori e ARB). Non vi sono, invece, problemi con l'assunzione di acido acetilsalicilico, che, anzi, riduce il rischio di pre-eclampsia. È importantissimo, per un decorso sicuro, l'accurato controllo della pressione sanguigna: circa il $16 \%$ delle donne con ADPKD va incontro a ipertensione, per la prima volta, in gravidanza, spe- cialmente negli ultimi mesi di gestazione.

Se la funzione renale non è compromessa in modo importante, la gravidanza non è motivo di peggioramento funzionale e può, generalmente, essere portata a termine in modo sicuro, ma è indispensabile un assiduo controllo per evidenziare precocemente ogni complicanza: il rischio di progressione del danno renale può essere grave e aumenta, anche se in modo non strettamente correlabile e prevedibile, in relazione al deficit funzionale presente prima della gravidanza.

La pre-eclampsia ha una frequenza superiore del $18 \%$ rispetto alla popolazione generale, specialmente nei soggetti che erano ipertesi prima della gravidanza: è assai importante saper cogliere precocemente e segnalare sintomi specifici (alterazioni della vista, cefalea, vomito, dolore toracico improvviso), così come edemi da ritenzione e proteinuria. Una grave ipertensione o una pre-eclampsia possono essere il segno di un'alterazione funzionale placentare, specialmente quando l'alterazione della funzione renale è più grave; in questo caso, si possono avere distacchi placentari precoci, con sanguinamento, e il parto deve essere iniziato urgentemente. In altri casi, sarà la gravità della pre-eclampsia a dettare il momento migliore per il parto, tenendo conto dei rischi della donna gravida e del grado di prematurità del bambino; la maggior parte delle gravidanze che sviluppano pre-eclampsia consente, comunque, la nascita di un bambino in salute. Per la valutazione della funzione renale in gravidanza il GFR non è un parametro ben accurato; è preferibile, piuttosto, il semplice dosaggio della creatinina sierica. Non si è notato un accrescimento delle cisti renali in gravidanza, mentre è noto l'aumento volumetrico delle cisti epatiche, senza che questo sia, peraltro, accompagnato da un peggioramento della funzionalità renale o da alterazioni fetali. Il parto, che, generalmente, deve essere anticipato di qualche tempo, è possibile anche per via naturale e la scelta del cesareo deve essere valutata caso per caso.

Qol familiare. Analogamente ad altre malattie ereditarie per le quali le terapie sono carenti, la diagnosi di ADPKD può avere un'influenza psicologica altamente negativa anche sul benessere familiare: ciò può manifestarsi, dal punto psicologico, nelle comuni attività quotidiane. Non sono rari comportamenti negazionali, dettati dal timore del riconoscimento della patologia nei figli, che comportano, con la loro attuazione, ritardi diagnostici e un tardivo inizio delle procedure comportamentali e farmacologiche, con conseguente più rapida e grave compromissione patologica e funzionale. D'altra parte, la consapevolezza della malattia può comportare, da parte dei genitori, attenzioni superiori alle reali necessità, con atteggiamenti proibitivi anche di fronte ad attività fisiche del tutto compatibili con la patologia. Inoltre le molteplici manifestazioni patologiche connesse all'ADPKD richiedono periodici controlli clinici e, non raramente, il ricovero ospedaliero, con influenza negativa sulle dinamiche familiari. Con l'instaurarsi dell'IRC, poi, sono necessari periodici accessi presso Strutture Sanitarie o l'organizzazione domestica, per l'effettuazione della terapia sostitutiva. Infine, di fronte a un programma di tra- 
pianto, la presa in carico della famiglia è ancora più onerosa: l'ovvia preoccupazione per l'iter chirurgico e l'esordio della terapia immunosoppressiva e, ancora di più, nel caso di un eventuale programma di donazione renale da vivente, l'instaurarsi di complesse dinamiche interpersonali.

Un supporto psicologico e collaborativo può essere trovato, oltre che da parte dei Sanitari coinvolti (Medico di Medicina Generale o Specialisti Nefrologi), anche nelle Associazioni di Pazienti che, in molte parti del mondo, sono andate formandosi e il cui apporto conoscitivo e supportivo è diventato sempre più una risorsa fondamentale (Tab. II)

Nonostante I'ADPKD non sia più considerata una malattia rara, vi sono ancora molti aspetti che andrebbero chiariti e in merito a cui si avverte, anche da parte dei Nefrologi, la necessità di un ulteriore approfondimento culturale. Da un recente Panel, i cui risultati sono stati presentati al Congresso Nazionale della Società Italiana di Nefrologia del 2015, si evince che le carenze conoscitive più citate da parte degli Specialisti Nefrologi intervistati sono relative al controllo pressorio e all'utilizzo di antiipertensivi, ai criteri diagnostici in pazienti con familiarità, all'ADPKD in età pediatrica e in gravidanza, alle peculiarità specifiche della terapia sostitutiva, al trattamento delle complicanze, alla ricerca delle manifestazioni extrarenali e, infine, ai marcatori prognostici di progressione (28).

In generale, per i pazienti ADPKD, il sostegno nefrologico percepito e un approccio olistico da parte dei Nefrologi di riferimento sono fattori predittivi positivi importanti nella scelta terapeutica più appropriata; l'ascolto attivo e l'empatia per le problematiche psicoemozionali derivanti da preoccupazioni circa stile di vita, immagine corporea e alterazioni

TABELLA II - Principali Associazioni di Pazienti con ADPKD nel mondo

The Finnish Kidney and Liver Association, Finland

Association Polykystose France (A.P.K.F.), France

PKD Familiäre Zystennieren e.V, Germany

AIRP onlus - Associazione Italiana Rene Policistico, Italy

PKD Foundation, Japan

NVN Nierpatienten Vereniging Nederland, Netherlands

AIRG Asociación para la Información y la Investigación de las Enfermedades Renales Genéticas, Spain

Njurförbundet (Swedish Kidney Foundation), Sweden

PKD Switzerland, Switzerland

PDK International, Switzerland

PKD Charity, United Kingdom

PKD Australia, Australia

PKD Foundation, United States

PKD Foundation of Canada, Canada

PKD South Korea, South Korea della sessualità migliorano la compliance ai trattamenti, con conseguenti risultati decisamente migliori (29).

\section{Terapia dell'ADPKD negli stadi iniziali}

Nell'ADPKD, la frequenza di mutazioni "de novo" è relativamente bassa e sono possibili, con precise tecniche di imaging come la $\mathrm{MRI}$ in $3 \mathrm{D}$, una valutazione e una misurazione accurate nel tempo delle cisti e del flusso renale (associate alla progressione della malattia); in più, l'evoluzione è lenta: tutto ciò fa del policistico un candidato ottimale alla nefroprevenzione. Svariate strategie consentono un sostanziale incremento della durata di vita e della Qol: la diagnosi precoce e il trattamento dell'ipertensione, le modifiche dello stile di vita, il trattamento delle complicanze renali ed extrarenali, la gestione delle alterazioni correlate alla CKD, le migliorate tecniche di dialisi e il trapianto renale. Vi sono notevoli differenze nei vari Paesi circa la prevenzione, la valutazione e il trattamento della policistosi renale, ma non sono disponibili Linee Guida (LG) ampiamente accettate, anche se, recentemente, sono stati pubblicati i risultati ottenuti da un Panel di esperti e dai pazienti di 20 nazioni, che possono essere considerati la base di una prima stesura di LG condivise (30). Dopo la scoperta dei geni PKD1 (1994) e PKD2 (1996), la ricerca di base e traslazionale è aumentata esponenzialmente negli ultimi tre decenni, rendendo disponibile una diagnosi genetica molecolare. Molti obiettivi terapeutici sono stati identificati e testati in modelli animali e studi clinici hanno prodotto risultati incoraggianti, tendenti ad armonizzare e a standardizzare le terapie (30).

È ampiamente riconosciuta l'importanza della terapia antiipertensiva: in particolare lo studio HALT PKD del 2015 ha valutato i risultati degli ACE inibitori come prima linea di trattamento. Nel giovane con buona funzione renale, mantenendo la pressione a livelli inferiori a quelli raccomandati dalle LG, I'incremento volumetrico renale è rallentato, diminuiscono le resistenze vascolari, l'albuminuria e l'indice di massa ventricolare sinistra, inoltre si ha una minor riduzione del GFR (30).

Terapie comportamentali. I pazienti con IRC che ricevono una terapia medica nutrizionale (MNT) loro assegnata da un dietologo esperto e dedicato possono migliorare in modo importante lo stato nutrizionale e posticipare l'esordio del trattamento dialitico. Se il trattamento dietetico inizia agli stadi 3 o 4 di CKD, si ottiene una più lenta progressione a ESRD rispetto a chi imposta tale programma allo stadio 5 . Sono ridotte sia la progressione della malattia che le complicanze relative alla CKD, come Protein Energy Wasting, Electrolyte Imbalance, deficit vitaminici e alterazioni minerali ossee. In un recentissimo studio, De Wall ha dimostrato che specifici atteggiamenti terapeutici portano a un quadro ematologico migliore (tasso di albumina, fosforo, calcio e PTH) e a un minor declino del GFR, con tempo di esordio dialitico tre volte più rallentato (31). Come in tutte le CKD, l'educazione alimentare è, quindi, fondamentale fin dai primi stadi e, poi, per tutto il corso 
della malattia. Per controllare l'eccessiva assunzione di fosforo, occorre limitare gli alimenti che ne sono particolarmente ricchi, preferendo vegetali bolliti ed escludendo cibi con additivi contenenti fosforo (32). Questo elemento ha un ruolo notevole nell'insorgenza di alterazioni minerali e ossee (MBD): l'iperfosforemia si verifica quando l'assorbimento intestinale è decisamente superiore rispetto all'escrezione renale o alla rimozione con la dialisi; giova ricordare che il fosforo è il componente principale di diversi additivi (acido fosforico, fosfati e polifosfati) utilizzati nella trasformazione industriale per allungare i tempi di conservazione degli alimenti e migliorarne il colore o il sapore: è da tenere ben presente che la quantità di fosforo da conservanti è notevole rispetto al contenuto di fosforo naturale degli alimenti e che i sali di fosforo inorganici sono assorbiti in modo quasi totale.

Terapie farmacologiche. Con la migliore comprensione dei meccanismi che sono alla base della malattia, si sono identificati farmaci potenzialmente in grado, se non di curare, almeno di rallentare l'evoluzione della nefropatia da ADPKD; studi sperimentali e clinici si sono concentrati su 2 gruppi di molecole: 1) inibitori della proliferazione cellulare (agenti antiproliferativi) e 2) inibitori della secrezione dei liquidi (antagonisti della vasopressina e della somatostatina).

1) Tra i farmaci del primo gruppo, gli inibitori del recettore dell'EGF (Erb-B receptor tyrosine kinase, come Erb-B1 ed Erb-B2 tirosina chinasi, Src chinasi, MEK e cdk inibitori) hanno dimostrato un ruolo cruciale nella crescita cistica. Gli inibitori della tirosina chinasi, come Bosutinib (già utilizzato nella terapia di alcune neoplasie linfoproliferative e del carcinoma mammario) si sono dimostrati in grado, su modelli animali, di inibire l'ingrandimento delle cisti. Farmaci m-TOR inibitori (Sirolimus ed Everolimus) inibiscono la formazione di cisti e riducono il volume del rene in molti modelli animali: hanno, come target principale, la FK506 binding protein; questo legame destabilizza l'associazione tra mTOR e raptor (nota anche come complesso TORC1), che, in condizioni normali, induce la fosforilazione della chinasi p70S6, promuovendo, così, la crescita cellulare. Il risultato finale ottenibile, quindi, da questi farmaci è di inibire la crescita cellulare e di ridurre la produzione delle cellule e la loro risposta ai fattori di crescita endoteliali, i cui recettori sono espressi dai tessuti cistici (33). Sono in corso alcuni trial clinici per valutare la reale efficacia di questa classe di farmaci; il loro ruolo terapeutico nell'APKD è ancora incerto: è possibile che queste molecole possano rallentare la crescita volumetrica dei reni e il deterioramento funzionale progressivo in qualche sottogruppo di pazienti, ma potrebbero risultare inefficaci o, addirittura, dannosi in altri. Ulteriori trial dovranno essere promossi per identificare i potenziali responder, il livello di funzione renale ottimale per l'avvio del trattamento e, soprattutto, la dose ottimale di impiego (34). Il Sirolimus (SIR) è un immunosoppressore con forti effetti antiproliferativi, potenzialmente in grado di fermare o ridurre la crescita delle cisti e di mante- nere la funzione renale, aumentando in modo significativo la percentuale del volume parenchimale, almeno nel breve termine, come evidenziato dallo studio di Perico e Remuzzi (35). La somministrazione giornaliera determina una perdita degli effetti antiproliferativi sulle cellule tubulari renali, mentre la somministrazione settimanale può avere efficacia nel ridurre la crescita delle cisti e nel preservare la funzione renale, come sembra dimostrare lo studio in corso Vienna RAP Study (36).

Everolimus sembra ritardare la crescita volumetrica dei reni, ma non rallenta il decremento funzionale; inoltre, nell'esperienza di Walz et al., è riferito un alto grado di effetti collaterali (37).

2) Gli antagonisti della vasopressina: OPC-31260 e Tolvaptan sono molecole probabilmente in grado di ridurre la formazione delle cisti nei ratti. II razionale dell'utilizzo di un antagonista del recettore V2 della vasopressina e dell'arginina per rallentare la progressione del danno renale è rappresentato dalla sua capacità di ridurre i livelli di cAMP e, di conseguenza, la secrezione di fluido all'interno delle cisti (33).

L'octreotide, analogo della somatostatina, sembra inibire l'accumulo di cAMP nelle cisti renali ed epatiche; la somatostatina è un ormone prodotto a livello gastrointestinale e ha un'emivita inferiore ai 3 minuti, caratteristica che ne esclude l'utilizzo clinico; sostanze analoghe (come octreotide e lanreotide) presentano un'emivita più lunga (38). Le modalità d'azione della somatostatina e dei suoi analoghi sono vari: a) si legano al recettore SST2, SST3 e SST5 e inibiscono il segnale del CAMP nei colangiociti e nelle cellule epiteliali tubulari; il cAMP ha un ruolo fondamentale nella secrezione di fluido nelle cisti e nella stimolazione della proliferazione cellulare; b) bloccano la secretina, che rappresenta il principale agonista dell'adenilatociclasi nei colangiociti e che contribuisce, insieme all'ADH, alla modulazione della concentrazione delle urine; c) sopprimono l'IGF1, un fattore di crescita dell'endotelio vascolare, e altri fattori di crescita implicati nella cistogenesi (39). Gli analoghi della somatostatina sono abbastanza ben tollerati e già commercializzati per altre indicazioni: emorragie acute gastrointestinali, profilassi delle complicanze di interventi sul pancreas e trattamento di fistole pancreatiche, pancreatiti acute e chetoacidosi diabetica $(38,39)$.

II Tolvaptan è stato autorizzato alla commercializzazione in Europa per il trattamento dell'ADPKD dalla Commissione Europea nel Maggio del 2015; è stato preferito alla molecola inizialmente impiegata (OPC 31260) perché più potente e selettivo e non sembra poter inibire la crescita di cisti a livello epatico (non vi sono recettori). A breve termine (una settimana) riduce la filtrazione glomerulare, con possibile aumento della vasopressina ematica e lieve incremento dei valori di creatinina e riduzione del volume renale e del volume delle cisti e del peso corporeo (40). Anche a lungo termine, dopo 3 anni di trattamento, ha dimostrato minore crescita del volume delle cisti renali, decremento dell'aumento annuo del 
volume renale e migliore salvaguardia della funzione, dimostrata da un declino di GFR inferiore e più lento (41). Lo studio TEMPO 3-4, utilizzando i criteri del CRISP (Consortium for Radiologic Imaging Studies of Polycystic Kidney Disease) e fondandosi sul concetto ormai acquisito del declino funzionale in rapporto all'accrescimento volumetrico, ha dimostrato che la terapia a lungo termine ( 3 anni) con Tolvaptan rallenta la progressione del declino funzionale, ma non ne consente il recupero e non ha influenza sull'ipertensione. Fondamentale l'esordio terapeutico precoce: reni piccoli nel paziente giovane rispondono maggiormente alla terapia; d'altra parte, anche reni ormai di grosse dimensioni, ma con funzione ancora conservata, possono giovarsi della terapia. Tolvaptan, inoltre, risulta maggiormente efficace e con minori effetti collaterali rispetto a Sirolimus ed Everolimus (42). La quota di perdita funzionale è significativamente correlata al volume renale e all'età della diagnosi. La differenza di $\Delta$-GFR dovrebbe essere prevista almeno 10 anni prima dell'insorgenza dell'ESDR, per consentire una precoce identificazione dei soggetti a maggior rischio e l'esordio precoce della terapia (43).

Terapie chirurgiche. L'ADPKD necessita, per molti anni, di una terapia tipicamente internistica, generalmente a carico dello specialista Nefrologo. In alcune circostanze, richiede l'intervento dell'Urologo per il trattamento di complicanze come ostruzione della via escretrice da compressione, UTI recidivanti, processi di ascessualizzazione cistica, episodi di ematuria ripetuti o di grave entità, litiasi renale e sospetto di neoplasia (della via escretrice, parenchimale o intracistica). Lo specialista urologo prende parte, inoltre, al processo decisionale ed effettua, se necessaria, la nefrectomia per la creazione di un idoneo spazio addominale in vista del trapianto renale.

L'ostruzione della via escretrice da compressione cistica non è un'evenienza frequente e può esser trattata con puntura transcutanea e svuotamento delle cisti (44). Le punture di cisti multiple e la resezione del tetto (intervento di Rovsing), proposte ed eseguite in passato a cielo aperto, non sembrano migliorare la funzione renale né impedirne l'ulteriore deterioramento (45); sembrano poter fornire, invece, un sollievo a lungo termine della sintomatologia algica (46). Un anno dopo lo svuotamento e la decorticazione delle cisti si hanno anche un decremento pressorio e una migliore funzione renale, $\mathrm{ma}$, a 3 anni dall'intervento, la funzione renale torna ai valori precedenti all'intervento; inoltre, la tecnica non è esente da complicanze e le difficoltà chirurgiche sono maggiori (47). La marsupializzazione e la decorticazione di cisti sintomatiche per via laparoscopica, attuate su piccoli numeri di pazienti, lasciano anch'esse adito a dubbi circa la corretta indicazione, specialmente in presenza di cisti infette, e, comunque, non si sono dimostrati risultati definitivi su dolore, ipertensione e mantenimento della funzione renale (48-50).

La TAE (embolizzazione arteriosa transcatetere con spirali) ha consentito una regressione volumetrica delle cisti sino al $53 \%$ a un anno: questo, secondo lo studio condotto da Ubara una decina di anni fa, consentirebbe anche un miglioramento della Qol e dello stato nutrizionale (51).

Numerose sono le situazioni patologiche che portano di necessità alla nefrecromia di bonifica. Come già riportato da Sing nel 1991, specialmente nel sesso femminile, le UTI recidivanti, con possibile ascessualizzazione cistica, sono la complicanze più frequenti, di difficile trattamento per la bassa penetranza degli antibiotici all'interno delle cisti infette, e, in dialisi, sono la prima causa di morte (39.8\%) (52). Le gravi emorragie (all'interno delle cisti, a livello perirenale o nella via escretrice), costituiscono la seconda causa renale di morte in dialisi (38.8\%) (10) e richiedono la nefrectomia: in questi casi, l'embolizzazione selettiva transcutanea (della cisti sanguinante o dell'arteria renale) consente di ottenere una stabilizzazione emodinamica, alla quale far seguire la nefrectomia con rischi minori $(5,53)$. La neoplasia renale insorta nel contesto di una policistosi ha una frequenza intorno all'1$2 \%(54)$, anche se alcuni Autori hanno riportato percentuali decisamente superiori (24.1\%) (55). Benché la nefrectomia parziale sia proponibile in rari casi di bilateralità lesionale, la nefrectomia mono o bilaterale costituisce, di norma, il percorso obbligato di trattamento.

\section{Trattamento sostitutivo dell'ADPKD nell'ESRD}

Emodialisi. La sopravvivenza dei pazienti con ADPKD in dialisi sembra essere maggiore di quella dei pazienti affetti da altre nefropatie: ciò, probabilmente, è correlato a una minore incidenza di coronaropatia (età media inferiore) e a livelli medi di EPO e di emoglobina più elevati (56).

In emodialisi, generalmente, le condizioni emodinamiche sono migliori che nei pazienti non ADPKD, ma presentano un maggior rischio teorico di complicanze: in circa il $50 \%$ dei casi sono frequenti le UTI (che possono interessare vescica, parenchima renale e strutture circostanti del retro peritoneo), ematuria ed emorragia intracistica o perirenale. In dialisi, le complicanze epatiche sono dovute all'aumento numerico e volumetrico delle cisti, specialmente nel sesso femminile con possibili infezione, emorragia od ostruzione dei vasi circostanti. Le complicanze cardiovascolari (come le anomalie valvolari) non contribuiscono significativamente alla mortalità e alla morbilità cardiaca nel paziente ADPKD in dialisi rispetto al non dializzato e l'infarto è meno frequente nei pazienti in emodialisi, indipendentemente dall'ipertrofia ventricolare e dai parametri relativi all'assetto lipidico, mentre l'ipertensione è molto frequente (56).

Dialisi peritoneale. In passato le indicazioni alla dialisi peritoneale (PD) nei pazienti con ADPKD erano limitate per il timore che lo spazio intraddominale, necessario per gli scambi peritoneali, potesse essere non sufficiente: si aveva la convinzione che i grossi organi addominali, riducendo l'area effettiva di scambio e aumentando ulteriormente la pressione intraddominale, avrebbero potuto inficiare gli scambi peritoneali e aumentare il rischio di ernie o di idrotorace. Si temeva che 
la frequente presenza di diverticoli intestinali e la non rara infezione delle cisti potessero incrementare il rischio di peritoniti, indotte soprattutto da microrganismi G-. Esistono, però, alcuni studi $(43,57)$ che dimostrano che sopravvivenza e incidenza di peritonite sono analoghe a quelle dei pazienti non diabetici e non policistici, mentre è realmente maggiore il rischio di ernie addominali. Oggi, la PD in corso di ESRD da ADPKD è considerata una valida opzione di trattamento che consente una sopravvivenza simile a quella dei soggetti in emodialisi (58).

L'alta percentuale di pazienti con progressione di CKD endstage costituisce, per il sistema sanitario pubblico e privato, un motivo di interesse, al fine di comprenderne le caratteristiche e di indagarne i costi. Nei pazienti ADPKD, il costo totale di assistenza sanitaria è inferiore rispetto a quello di altri pazienti in dialisi perché sono generalmente più giovani; ciò comporta che gli interventi terapeutici in grado di prevenire o di ritardare la progressione verso I'ESRD hanno un duplice potenziale positivo: aumentano il tempo di vita senza necessità dialitiche e riducono i costi di assistenza. I pazienti con policistosi, inoltre, hanno un inferiore Charlson Comorbidity Index e un minor tasso di comorbilità (diabete, insufficienza cardiaca, malattia coronarica e malattia cerebrovascolare). La spesa sanitaria, ovviamente, cresce in modo decisivo con la progressione patologica a ESRD; anche in questo caso, però, c'è da notare che, generalmente, i costi sono più contenuti (59), infatti il paziente ADPKD ha spesso un lasso di tempo più lungo per prepararsi alla dialisi e, in considerazione della natura familiare della malattia, una maggiore esperienza diretta della terapia sostitutiva, che, sovente, inizia già con una fistola artero-venosa pre-confezionata e non con un catetere venoso centrale (CVC), oppure effettua, come prima modalità, la dialisi peritoneale.

\section{Trapianto}

Trapianto renale. L'ADPKD rappresenta, in Francia, il 15\% di tutti i trapianti renali e si situa al terzo posto come patologia motivo di grafting (60). Il policistico è un ottimo candidato: la malattia, infatti, non recidiva nel rene trapiantato e la sopravvivenza del paziente e dell'organo non risultano mai inferiori a quelle dei pazienti sottoposti al trapianto renale per altra patologia, anche se, rispetto a questi, si ha un maggiore rischio di complicanze post-trapianto, come eritrocitosi e trombo embolia, dismetabolismi, aneurismi sintomatici e ipertensione arteriosa. Sono anche più frequenti diverticolosi e diverticolite del colon-sigma e, in generale, patologie gastrointestinali che richiedono un intervento chirurgico dopo il trapianto: I'attenzione alle specifiche complicanze è fondamentale al fine di ottimizzarne il risultato globale (61).

Il rischio di rigetto, invece, è analogo o addirittura inferiore a quello dei pazienti non policistici e sicuramente minore di quello che grava su pazienti con nefropatie immunomediate (62). Alcuni studi (63) sostengono che ci sia un rischio di UTI incrementato dopo trapianto, mentre altri Autori (64) smen- tiscono questo dato.

L'insorgenza di diabete mellito è associata a un significativo impatto sulla Qol ed è causa, a lungo termine, di un aumento di morbilità e mortalità. De Mattos, nel 2005, valutò tale evento in pazienti sottoposti a trapianto renale e descrisse una significativa associazione tra ADPKD e sviluppo di diabete tipo II nel primo anno, dato 3 volte superiore a quello dei pazienti sottoposti a grafting per altre cause, con un evidente impatto negativo sulla sopravvivenza. II motivo di questa associazione non è chiaro e necessita di ulteriori indagini (65). È stato riportato anche un aumentato rischio di neoplasie cutanee non-melanoma, ma altri lavori hanno smentito questo dato, così come hanno negato anche la maggiore incidenza di diabete "de novo" (66).

Dopo il trapianto, il volume dei reni nativi diminuisce rapidamente nel primo periodo, poi più lentamente; a livello epatico, invece, le cisti tendono a crescere (può rendersi necessario un trattamento con un analogo della somatostatina), mentre gli eventi cerebrovascolari sono rari, con un effetto marginale su morbilità e mortalità. Mosconi et al. hanno analizzato i risultati italiani del trapianto di rene in ADPKD negli anni 2002-2010; evidente la migliore sopravvivenza del graft rispetto ad altre patologie renali ( $86 \%$ vs $82 \%$ a 5 anni), con un analogo tasso di mortalità ( $92 \%$ vs $79 \%$ a 1 anno). Per quanto riguarda la nefrectomia prima del trapianto, gli Autori notarono un atteggiamento conservatore in 32/35 Centri Trapianto (la principale indicazione riferita era la mancanza di spazio addominale); anche nell'esperienza italiana, quindi, con una corretta gestione, il paziente policistico si è dimostrato un ottimo candidato al trapianto di rene (67).

Trapianto combinato fegato-rene. Nei pazienti ADPKD in ESRD e con fegato di grandi dimensioni, anche se ben funzionante (l'insufficienza epatica è una rara indicazione al trapianto epatico), occorre considerare la possibilità di un trapianto combinato fegato-rene. La decisione è collegiale, coinvolge Chirurghi e Nefrologi e riguarda la necessità di un trapianto renale, al quale si associa una funzione epatica in possibile evoluzione sfavorevole in termini di complicanze quoad vitam (infezioni, sanguinamento, ipertensione portale, ecc.) o quoad valetudinem (anoressia, grave dispepsia, malnutrizione, astenia, limitazione dell'attività fisica o lavorativa, dolore, ecc.). Principale indicazione al trapianto combinato è, quindi, la grave epatomegalia: i pazienti traggono notevole beneficio dalla risoluzione dell'iperdistensione addominale e dell'anoressia (68). La carenza di organi, però, rende complesso e non routinario il programma di un trapianto combinato, che rimane disponibile solo per pazienti altamente selezionati, anche se si è dimostrato essere una scelta eccellente per il notevole miglioramento della Qol, mentre morbilità e tasso di sopravvivenza a 1 e a 2 anni sono del tutto simili al trapianto combinato effettuato con altre indicazioni (69).

I vantaggi del trapianto combinato fegato-rene sono rappresentati dalla risoluzione della maggior parte dei problemi clinici con sospensione della dialisi e sostituzione funzionale 
dei due organi; inoltre, la bassa incidenza di IRC dopo trapianto epatico comporta riduzione di morbilità e mortalità e minor numero di ricoveri ospedalieri nel tempo (62). A lungo termine, la sopravvivenza del rene trapiantato è più alta nei soggetti sottoposti a trapianto combinato che in quelli sottoposti al trapianto del solo rene e, analogamente al trapianto combinato fegato-cuore, è valida l'ipotesi che il fegato possieda una capacità di "protezione" del rene trapianto simultaneamente (70). Negli ultimi anni, l'introduzione del Meld score nella valutazione epatica pre-trapianto ha consentito un incremento dei trapianti combinati fegato-rene, fornendo un'allocazione equa e corretta, che tiene in giusto conto la realtà della domanda superiore all'offerta (71).

Nefrectomia di bonifica. In vista di un trapianto renale, risulta imperativa la creazione di un idoneo spazio addominale, ma "storicamente" e sino a oggi, sono state presenti due opposte correnti di pensiero riguardo all'effettuazione di una nefrectomia di bonifica (mono o bilaterale) routinaria.

1) Nefrectomia di routine. Molti Autori, nel tempo, hanno sostenuto l'opportunità di una nefrectomia (mono o bilaterale) da eseguire precedentemente al trapianto o in sua concomitanza.

La binefrectomia può esser motivata da ripetute emorragie o infezioni renali bilaterali: reni estremamente voluminosi possono essere causa di dolore, dispnea e disturbi gastrointestinali e, al momento del trapianto, possono interferire con l'allocamento del graft nello spazio pelvico, come già ebbe modo di sostenere nel 1991 Rayner, che riferì, seguendo questo approccio, un decremento della mortalità e della morbilità da cause settiche dopo trapianto (72). Secondo Glassman et al., l'esecuzione di una nefrectomia bilaterale concomitante al trapianto renale non è causa di più alte morbilità o mortalità; è, al contrario, evidente un maggior grado di soddisfazione da parte dei pazienti, mentre i soggetti non sottoposti a procedura concomitante, a parere degli Autori, avrebbero fortemente desiderato che fosse loro offerta questa opzione. La nefrectomia bilaterale può fornire maggiore spazio per il posizionamento del rene da trapiantare e alleviare i sintomi nel postoperatorio. Eseguita dopo il trapianto, invece, si è dimostrata causa di un maggior numero di complicanze (73). Anche Tabibi si schiera tra i fautori della nefrectomia (mono o bilaterale) al momento del trapianto: l'asportazione bilaterale dei reni nativi può essere effettuata anche precedentemente al trapianto, al prezzo, però, di anuria e di inevitabili alterazioni metaboliche; eseguirla come prima fase del trapianto comporta una migliore accettazione e con scarsa morbilità (74).

Per Fuller et al., è preferibile la nefrectomia mono o bilaterale al momento del trapianto, specialmente in caso di donazione da vivente (75); anche nell'esperienza di Wagner et al., in considerazione anche dei molteplici sintomi sistemici in corso di ADPKD, con un evidente decremento della Qol, effettuare la mono o binefrectomia concomitante al trapianto appare sicuro, specialmente in caso di trapianto da donatore vivente (76). Anche altri Autori, per i molteplici sintomi sistemici e l'evidente decremento della Qol, ritengono utile la mono o binefrectomia in concomitanza del trapianto, specialmente in caso di riceventi da donatore vivente (77). Whitten et al. (78) la eseguono abitualmente in pazienti con ESRD e dolore addominale cronico, sostenendo l'utilità della binefrectomia in tempo unico per limitare il numero di interventi e per evitare che il dolore, per la crescita del rene residuo, possa ripresentarsi. Fondandosi sull'esperienza personale di 159 trapianti in pazienti ADPKD, Nunes sostiene che la nefrectomia monolaterale può essere eseguita in modo sicuro al momento del trapianto, senza ripercussioni sulla durata della degenza ospedaliera, sulla funzione (a breve e a lungo termine) del graft e sulla sopravvivenza. Questa scelta comporta, però, a parere dell'Autore, un tempo operatorio più lungo e il ricorso a un maggior numero di trasfusioni (79). Anche Sulikowski et al. consigliano di effettuare di routine la nefrectomia (mono o bilaterale) prima del trapianto oppure la binefrectomia in corso di trapianto, del quale riferiscono, con questo comportamento, un minor numero di fallimenti e di complicanze (80). Lucas et al. sostengono che la mononefrectomia omolaterale al momento del trapianto non dimostra una significativa morbilità rispetto al solo trapianto renale (81) e, dopo 100 trapianti in ADPKD, anche Neff conclude che è una pratica consigliabile, scevra da mortalità, anche se gravata da un alto tasso di complicanze, con necessità di revisione chirurgica del $12 \%$ dei casi (82).

Negli ultimi anni, è stata proposta la tecnica laparoscopica: Mak et al., se è necessario creare spazio per il graft, effettuano la binefrectomia pre-trapianto con tecnica hand-assisted (83); anche Lee sostiene la nefrectomia transperitoneale laparoscopica hand-assisted e consiglia, per non ampliare l'incisione di servizio, di procedere allo svuotamento delle cisti prima dell'estrazione del rene devascolarizzato (84). Per reni particolarmente voluminosi, pur con grande esperienza specifica, Lipke segnala, in corso di nefrectomia laparoscopica, un'alta percentuale di conversioni in open e consiglia, quindi, di iniziare l'intervento comunque a cielo aperto (85).

Secondo Shumate et al., la nefrectomia omolaterale concomitante è associata a una significativa riduzione della dose di farmaci necessari per il controllo dell'ipertensione; inoltre, la nefrectomia controlaterale, effettuata dopo il trapianto, consente un controllo ancora migliore della pressione ematica (86). Altri Autori preferiscono la bonifica prima del trapianto, per evitare di prolungare i tempi anestesiologici del grafting, ridurre le perdite ematiche ed evitare altre possibili complicanze, relative al concomitante esordio della terapia immunosoppressiva (87). La nefrectomia bilaterale, anche in corso di trapianto da donatore vivente, non ha, nell'esperienza di Skauby et al., un impatto negativo sul paziente né sulla sopravvivenza del graft; il tempo di ricovero è, però, più lungo e le complicanze sono maggiori, anche con il rischio di perdita del rene: è, quindi, necessaria, a parere degli Autori, al momento della scelta programmatica, una specifica infor- 
mazione al paziente (88)

Desai et al. hanno utilizzato la tecnica laparoscopica per la routinaria bonifica pre-trapianto: sottolineano l'importanza di un'accurata dissezione chirurgica dei piani, per evitare rotture di cisti, causa, nel postoperatorio, di ileo paralitico e di morbilità aumentata (89). L'approccio laparoscopico transperitoneale è sostenuto da Verhoest et al., che riportano, però, un alto tasso di complicanze (33\%), analogamente a quello retroperitoneale. Per grandi masse renali, la via transperitoneale risulta, nella loro esperienza, comunque più agevole, per lo spazio operativo maggiore, e l'asportazione del rene deve essere preceduta da morcellazione. In ogni caso, la nefrectomia pre trapianto laparoscopica è causa di un ritardo temporale inferiore, rispetto alla via open (circa 3 mesi contro 12), dell'intervento di grafting (90). L'analisi sistematica della Letteratura circa la nefrectomia laparoscopica per ADPKD, proposta da Williamson et al. nel 2014 (91), ha considerato 15 studi retrospettivi (non disponibili studi prospettici randomizzati) per un totale di soli 293 pazienti. La via transperitoneale risulta la più utilizzata, ma sono contraddittori i dati circa tempi chirurgici (variabili da 90 a 568 minuti), numero di conversioni, durata della degenza (da 2.6 a 11 giorni) e complicanze intra e postoperatorie (rispettivamente 24 e 68).

Anche recentemente, sono stati riportati buoni risultati per la binefrectomia in corso di trapianto, approccio che consente di evitare il periodo dialitico e di diminuire il costo dovuto agli interventi seriati, con sopravvivenza del graft, soddisfazione del paziente e morbilità accettabili (92).

2) Nefrectomia di bonifica non routinaria ma solo in caso di reale necessità, in previsione di trapianto. La scelta di questo iter comportamentale deriva dalla considerazione che il processo patologico è bilaterale e che, quindi, l'economia del parenchima deve essere la regola: anche in dialisi, una seppur minima funzione renale riduce l'anemia, l'osteodistrofia, la ritenzione di fluidi e l'iperkaliemia; inoltre, mantenere una diuresi presente e congrua preserva la funzionalità vescicale in vista del trapianto renale. Già nel 1996, Knispel et al. pubblicarono, con tale condotta, risultati positivi del trapianto (93) e, anni dopo, Muller et al., nel 2005, fornirono dati che non dimostravano alcuna differenza sull'esito del trapianto in presenza di entrambi i reni nativi (94).

A parere di molti Autori è opportuno, quindi, mettere in atto un approccio individualizzato, che tenga in considerazione le comorbilità, le complicanze e la sintomatologia specifica relative alle cisti (95). A parere di Cohen et al., con esperienza su 146 soggetti ADPKD sottoposti a trapianto renale, la nefrectomia sistematica unilaterale o bilaterale non deve essere proposta, per evitare le complicanze dello stato anefrico: è preferibile aspettare, quando possibile, fino a quando il paziente non viene posto in dialisi; d'altra parte, lo sviluppo del trapianto pre-emptive ripropone il problema della nefrectomia concomitante al trapianto e, solo in questo caso, può essere una soluzione praticabile (96). Anche per Patel et al. non è utile procedere di routine alla nefrectomia preventiva, ma

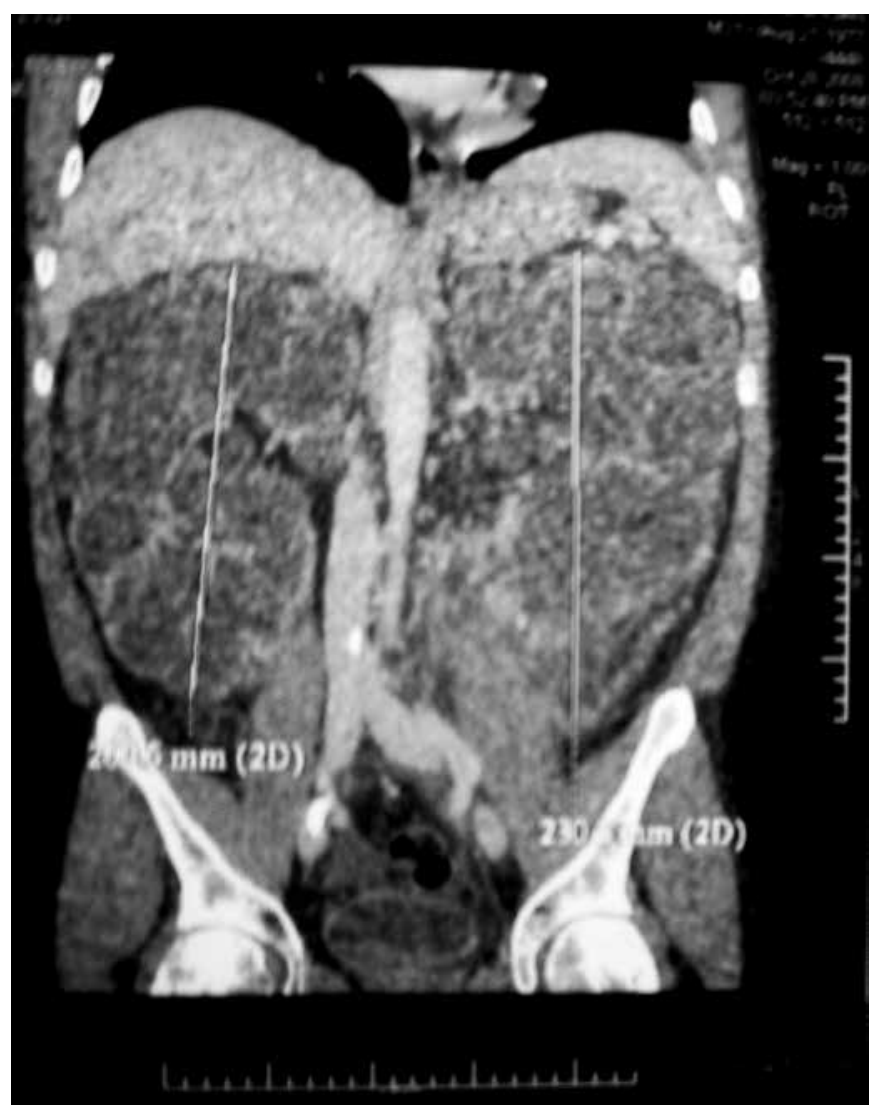

Fig. 2 - TC: reni policistici che occupano lo spazio pelvico.

solo in casi selezionati, come: insufficiente spazio per il graft, grave dolore o ripetuti episodi settici. In questi casi, è possibile l'accesso laparoscopico, ma se, dopo trapianto, si rende necessaria la nefrectomia controlaterale, questa è gravata da un tasso maggiore di complicanze (97).

Anche nella nostra esperienza, la nefrectomia di bonifica pre trapianto non è considerata necessaria di routine, ma è effettuata unicamente se sono presenti complicanze o il sospetto di neoplasia, così come per creare uno spazio pelvico idoneo quando quello esistente, valutato con TC o MRI (Fig. 2), è insufficiente all'allocazione del graft. Si è soliti eseguire la nefrectomia sinistra di routine, invece, in previsione di un trapianto combinato fegato-rene. Pur essendo ampia l'esperienza laparoscopica nel Centro di Torino, si è scelta sempre la via retroperitoneale open, riservando la transperitoneale a casi eccezionali o per la binefrectomia. Al fine di minimizzare le complicanze di parete relative all'accesso per via lombotomica (ampia cicatrice e asportazione parziale dell' $11^{\mathrm{a}}$ costa), negli ultimi anni si è avviata una tecnica mininvasiva, che prevede un'incisione lombare di soli $10 \mathrm{~cm}$ senza resezione costale, isolamento, svuotamento geometrico mirato di cisti non infette con ago collegato all'aspiratore, legatura del peduncolo vascolare sotto vista e asportazione del rene rimodellato attraverso l'incisione di minima. Questa tecnica si 
è rivelata sicura e ha dato buoni risultati, non ha prolungato in modo sensibile i tempi chirurgici, né è stata gravata da un tasso di complicanze superiore alla tecnica open tradizionale; nei confronti della tecnica laparoscopica ha costi evidentemente inferiori, non essendo necessari materiali operatori particolari (98).

La donazione di rene da parte di un soggetto appartenente a una famiglia ADPKD. Nel caso in cui si renda disponibile un possibile donatore di rene proveniente da una famiglia ADPKD con genotipo non noto, sono stati proposti dei criteri ecografici di esclusione della malattia: 1) se i reni risultano normali o presentano al massimo una cisti, in un soggetto che abbia un'età $>40$ anni, il VPN dell'ecografia risulta del $100 \%$; 2) nei soggetti di età compresa tra i 30 e i 39 anni l'assenza di cisti renali prova l'esclusione della malattia con un tasso di falsi negativi dello $0.7 \%$; 3 ) nei soggetti di età $<30$ anni, se l'esame dà un risultato negativo o indeterminato, I'utilità dell'ecografia per l'esclusione della malattia è scarsa e non deve essere utilizzata perché i falsi negativi sono circa il 4\%; 4) nei soggetti di 40-60 anni, l'assenza di almeno 2 cisti in ciascun rene ha un valore predittivo negativo del $100 \%$ e il criterio ecografico può essere utilizzato per l'esclusione della malattia.

\section{Considerazioni}

La malattia policistica renale è responsabile di circa il 4-6\% di tutte le IRC terminali in Italia. La terapia comportamentale e dietetica è in grado di rallentare, unitamente alla terapia farmacologica, l'evoluzione patologica verso l'IRC e la necessità di una terapia sostitutiva. Nessun farmaco, a oggi, è risultato di consolidata efficacia nel trattamento della malattia, anche se il Tolvaptan ha dimostrato a 3 anni una riduzione dell'incremento volumetrico dei reni, della perdita funzionale, del dolore cronico e degli eventi acuti. Sono assolutamente importanti, durante le fasi evolutive della malattia, il controllo delle complicanze e il trattamento mirato della sintomatologia presentata; in particolare la gestione dell'ipertensione, dell'ingombro addominale e del dolore. Numerose sono le possibilità terapeutiche farmacologiche e mininvasive che possono rendere più affrontabile e meglio sopportabili le conseguenze delle alterazioni anatomiche e funzionali dei singoli organi coinvolti. La Qol in corso di ADPKD risulta deteriorata sia a livello individuale che familiare: devono essere sempre tenuti presenti le restrizioni dietetiche e comportamentali, le limitazioni e i rischi per la donna in età fertile di intraprendere una gravidanza, le terapie impegnative, il timore e la necessità di ripetuti ricoveri ospedalieri per la gestione delle complicanze e le paure di progressione patologica (con conseguente inevitabile deterioramento funzionale e necessità di terapia sostitutiva) e di trasmissione alla prole dell'alterazione genetica.

Il complesso contesto psicopatologico, nelle sue interezza, peculiarità e cronicità, deve essere preso in carico in ogni fase della malattia dal Medico di Medicina Generale e, ancora di più, dallo specialista Nefrologo di riferimento, estendendo, a seconda dei momenti e delle necessità, ad altri Specialisti la presa in carico delle specifiche esigenze. È, inoltre, molto importante, in ogni fase della malattia, il ruolo di supporto che i familiari e le Associazioni di Pazienti hanno nell'affrontare i problemi quotidiani, che possono essere motivo di grave decremento della qualità di vita.

Quando la malattia raggiunge limiti di funzionalità renale talmente alterati da richiedere la terapia sostitutiva, la dialisi può essere programmata per tempo e possono essere predisposti i mezzi tecnici più idonei ad affrontarla in sicurezza e con minori disagi. Entrambe le modalità dialitiche (emodialisi e peritoneale) sono proponibili, efficaci e, generalmente, ben tollerate.

Il percorso del trapianto renale, oggi anche in fase preemptive, rimane comunque la scelta ottimale per la maggior parte dei pazienti, con risultati a lungo termine decisamente positivi. Può essere proposto un trapianto di solo rene per la risoluzione del problema funzionale, ma è altrettanto valida l'opzione di un trapianto combinato fegato-rene per migliorare la qualità di vita in caso di epatomegalia altamente sintomatica.

Rimane aperto il dibattito circa l'opportunità della nefrectomia di routine (mono o bilaterale pre trapianto o concomitante al trapianto). In Letteratura sono presenti esponenti di entrambe le posizioni: i fautori di tale approccio riferiscono che non ci sono complicanze aggiuntive, ma, piuttosto, c'è un miglior esito del trapianto, essendo ridotto il rischio di complicanze settiche e di compressione del graft. Sottolineano che circa il $43 \%$ dei casi non sottoposti a bonifica routinaria prima del trapianto richiede, comunque, la nefrectomia dopo il trapianto. Molti segnalano un maggior numero di complicanze e di perdita del graft nei pazienti non sottoposti a nefrectomia prima del trapianto.

I fautori, invece, della nefrectomia effettuata solo in caso di necessità (creazione dello spazio addominale o trattamento delle complicanze) rimarcano come, senza nefrectomia preventiva di routine, la sopravvivenza a 1 e a 5 anni non è inferiore, ricordano che il volume renale, dopo trapianto, tende a regredire sino al $40 \%$ dopo 3 anni e rimarcano l'importanza di rispettare la massa nefronica presente, anche se deficitaria, al fine di procrastinare al massimo la necessità di terapia sostitutiva e di limitare le alterazioni che la condizione di anefricità comporta.

Infine, quando si programma una nefrectomia per ADPKD, è imperativo che essa sia condotta con criteri personalizzati e mininvasivi, scegliendo, di norma, la via retroperitoneale per risparmiare l'integrità della cavità peritoneale e ricorrendo alla via transperitoneale solo in caso di binefrectomia; questo al fine di non alterare la cavità peritoneale (la cui integrità è una garanzia in caso di ulteriori necessità dialitiche) e di minimizzare il rischio di laparocele e di ernia parietale, peraltro decisamente frequenti in questi pazienti. 
Nel corso degli anni, sono riportati da tutti gli Autori risultati positivi nel trattamento dell'ESDR da ADPKD con il trapianto renale in tutte le modalità: da donatore deceduto $\mathrm{e}$ vivente, di singolo rene e combinato fegato-rene. La terapia immunosoppressiva non differisce dai pattern utilizzati per trapianti eseguiti per IRC da altre cause patologiche, ma, nella patologia policistica, può usufruire, in modo utilmente specifico, di farmaci mTOR inibitori.

Nella comprensione della malattia, così come nella messa in opera di strumenti educazionali, nelle nuove terapie e nel miglioramento delle politiche sanitarie rivestono un ruolo fondamentale le organizzazioni dei pazienti di cui esiste una fitta rete internazionale.

L'approccio multidisciplinare presso Centri specificatamente dedicati o con un interesse specifico per l'ADPKD è garanzia dell'ottimale presa in carico globale del paziente, che ne ricava maggiori sicurezze e benefici.

\section{Disclosures}

Financial support: No financial support was received for this submission. Conflict of interest: F.L. Otsuka Pharmaceutical Italy S.r.l. consultant.

\section{Bibliografia}

1. Torres V, Grantham JJ. Cystic diseases of the kidney. In Brenner B. editor. The kidney, Vol 6. Saunders Elsevier; Philadelphia 2008:1428-62.

2. Torres VE, Harris PC. Autosomal dominant polycystic kideney disease: the last 3 years. Kidney Int 2009;76:140-68.

3. US Renal Data System. Excerpts from the USRDS 2004. Annual Data Report. Am J Kidney Dis 2005;45(Suppl.):S1-280,

4. Gabow P. Autosomal dominant polycystic kidney disease. N Engl J Med 1993;329:332-42.

5. Braun WE. Autosomal dominant polycystic kidney disease: emerging concepts of pathogenesis and new treatments. CJM 2009;76(2):97-104.

6. Bajwa ZH, Sial KA, Malik AB, Steinman TI. Pain patterns in patients with polycystic kidney disease. Kidney Int 2004;66:1561-9.

7. McHugh K, Stringer D, Hebert D, Babiak C. Simple renal cysts in children: Diagnosis and follow-up with US. Radiology 1991; 178:383-5.

8. Torres VE, Grantham JJ. Cystic Diseases of the Kidney. IN: Brenner B.M. THE KIDNEY Ed. Elsevier 2007;41:1428-43.

9. Simms RJ, Travis DL, Durkie M, Wilson G, Dalton A, Ong AC. Genetic testing in the assessment of living related kidney donors at risk of autosomal dominant polycystic kidney disease. Transplantation 2015;99(5):1023-9.

10. Grantham LL, Chapman AB, Torres VE. Volume Progression in Autosomal Dominant Polycystic Kidney Disease: The Major Factor Determining Clinical Outcomes. Clin J Am Soc Nephrol 2006;1:148-57.

11. Dalgaard OZ. Bilateral polycystic disease of the kidneys. Acta Med Scand 1957;328:1-255.

12. Thomsen HS, Madsen JK, Thaysen JH, Damgaard-Petersen $\mathrm{K}$. Volume of polycystic kidneys during reduction of renal function. Urol Radiol 1981;3:85-9.

13. Walz G, Budde K, Manna M, et al. Everolimus in Patients with Autosomal Dominant Polycystic Kidney Disease. N Engl J Med 2010;363:830-40.
14. Ju W, Nair V, Smith S, Kretzler M et al. Tissue transcriptomedriven identification of epidermal growth factor as a cronic kidney disease biomarker. Sci Transl Med 2015:7(316):193.

15. Poppe C, Crombez G, Hanoulle I, Vogelaers D, Petrovic M. Improving quality of life in patients with chronic kidney disease: influence of acceptance and personality. Nephrol Dial Transplant 2013;28:116-21.

16. Valderrabano F, Jofre R, Lopez-Gomez JM. Quality of life in endstage renal disease patients. Am J Kidney Dis 2001;38:443-64.

17. Wasserfallen J, Halabi G, Saudan P, et al. Quality of life on chronic dialysis: comparison between haemodialysis and peritoneal dialysis. Nephrol Dial Transplant 2004;19:1594-9.

18. Tsai YC, Hung CC, Hwang SJ, et al. Quality of life predicts risks of end-stage renal disease and mortality in patients with chronic kidney disease. Nephrol Dial Transplant 2010;25:1621-6.

19. Finkelstein $F$, Wuerth $D$, Finkelstein $S$. Health related quality of life and the CKD patient: challenges for the nephrology community. Kidney Int 2009;76:946-52.

20. Kovac J, Patel S, Peterson R, et al. Patient satisfaction with care and behavioural compliance in end-stage renal disease patients treated with haemodialysis. Am J Kidney Disease 2002;39:1236-44.

21. Rizk D, Jurkovitz C, Veledar E, et al. Quality of Life in Autosomal Dominant Polycystic Kidney Disease Patients not yet on Dialysis. Clin J Am Soc Nephrol 2009;4:560-6.

22. Miskulin DC, Abebe KZ, Chapman AB, et al. Health related quality of life in patients with autosomal dominant polycystic kidney disease and CKD stages 1-4: a cross sectional study. Am J Kidney Dis 2014;63(2):214-26.

23. Baiwa ZH, Sial KA, Malik AB, Steinman TI. Pain patterns in patients with polycystic kidney disease. Kidney Int 2004;66: 1561-9.

24. Bourquia A. Autosomal dominant polycystic kidney disease (ADPKD) in Morocco. Multicenter study about 308 families. Nephrologie 2002;23:93-6.

25. Purnell TS, Auguste P, Crews DC, et al. Comparison of Life Participation Activities Among Adults Treated by Hemodialysis, Peritoneal Dialysis, and Kidney Transplantation: A Systematic Review. Am J Kidney Dis 2013;62(5):953-73.

26. Finkelstein $F$, Wuerth $D$, Finkelstein $S$. Health related quality of life and the CKD patient: challenges for the nephrology community. Kidney Int 2009;76:946-52.

27. Tsai YC, Hung CC, Hwang SJ et al. Quality of life predicts risks of end-stage renal disease and mortality in patients with chronic kidney disease. Nephrol Dial Transplant 2010;25:1621-6.

28. Capasso GB, Ferrara C, Galliani M, et al. Identificazione delle carenze conoscitive nell'ADPKD: risultati di una survey italiana. Abstract Book $56^{\circ}$ Congr SIN 2015; 501 POA; G Ital Nefrol 2015;32(S65).

29. Kovac J, Patel S, Peterson R et al. Patient satisfaction with care and behavioural compliance in end-stage renal disease patients treated with haemodialysis. Am J Kidney Dis 2002;39: 1236-44.

30. Chapman A, Devuyst O, et al. Autosomal-dominant polycystic kidney disease (ADPKD): executive summary from a Kidney Disease: Improving Global Outcomes (KDIGO) Controversies Conference. Kidney Int 2015;88:17-27.

31. De Wall D, Heaslip E, Callas P. Medical Nutrition Therapy for Chronic Kidney Disease Improves Biomarkers and Slows Time to Dialysis. J Ren Nutr. 2015; 26:1-9.

32. D'Alessandro C, Piccoli GB, Cupisti A. The "phosphorus pyramid": a visual tool for dietary phosphate management in dialysis and CKD patients. BMC Nephrology 2015;16:9.

33. Belibi FA, Edelstein CE. Novel targets for the treatment of autosomal dominant polycystic kidney disease. Expert Opin 
Investig Drugs 2010;19(3):315-28.

34. Ponticelli C, Locatelli F. Autosomal dominant polycystic kidney disease and mTOR inhibitors: the narrow road between hope and disappointment. Nephrol Dial Transplant 2010; 25(12):3809-12.

35. Perico N, Antiga $L$, et al. Sirolimus therapy to halt the progression of ADPKD. J Am Soc Nephrol 2010;21:1031-40.

36. Riegersperger $M$, Herkner $H$, Sunder-Plassmann $G$. Pulsed oral sirolimus in advanced autosomal-dominant polycystic kidney disease (Vienna RAP Study): study protocol for a randomized controlled trial. Trials 2015;16:182.

37. Walz G, Budde K, Manna M, et al. Everolimus in Patients with Autosomal Dominant Polycystic Kidney Disease. N Engl J Med 2010;363:830-40.

38. Gevers TJ, Drenth JP. Somatostatin analogues for treatment of polycystic liver disease. Curr Opin Gastroenterol 2011;27(3): 294-300.

39. Hogan MC, Masyuk TV, Page LJ, et al. Randomized clinical trial of long-acting somatostatin for autosomal dominant polycystic kidney and liver disease. J Am Soc Nephrol 2010;21(6):1052-61.

40. Irazabal MV, Torres VE, Hogan MC, et al. Short-term effects of tolvaptan on renal function and volume in patients with autosomal dominant polycystic kidney disease. Kidney Int 2011;80:295-301.

41. Torres VE, Chapman AB, Devuyst O, et al. Tolvaptan in Patients with Autosomal Dominant Polycystic Kidney Disease. N Engl J Med 2012;367:2407-18.

42. Torres VE, Meijer E, Bae KT, et al. Rationale and design of the TEMPO (Tolvaptan Efficacy and Safety in Management of Autosomal Dominant Polycystic Kidney Disease and its Outcomes) 3-4 Study, Am J Kidney Dis 2011;57:692-9.

43. Thong KM, Ong AC. The natural history of autosomal dominant polycystic kidney disease: 30 year experience from a single center. QJM 2013;106(7):639-46.

44. Lue YB, Anderson EE, Harrison HT. The surgical management of polycystic renal disease. Gynecol Obstet 1966:122:45-7.

45. Milam JH, Magee JH, Bunts RC. Evalutation of surgical decompression polycystic kidneys by differential rental clearance. J Urol 1963;90:144-8.

46. Elzinga LW, Barry JM, Bennett WM. Surgical management of painful polycystic kidneys, Am J Kidney Dis 1993:22:532-5.

47. Quian X, Sheng X, Li R, et al. Which stage of ADPKD is more appropriate feor decortiction? A retrospective study of 137 patients from a single Clinic. PLoS One 2015;10(5):e0120696.

48. Dunn MD, Portis AJ, Naughton C, Shalhav A, Mc Dougall EM, Clayman RV. Laparoscopic cyst marsupialization in patients with autosomal dominant polycystic kidney disease. J Urol 2001;165(6 Pt. 1):1888-92.

49. Lee DI, Androni CR, Rehman J, et al. Laparoscopic cyst decortication in autosomal dominant polycystic kidney disease: impact on pain, hypertension, and renal function. J Endourol 2003;17(6);345-54.

50. Seshadri PA, Polin EC, Pace D, Schachta CM, Cadeddu MO, Mamazza J. Transperitoneal laparoscopic nephrectomy for giant polycystic kidneys: a case control study. Urology 2001; 58(1):23-7.

51. Ubara Y. New Therapeutic option for autosomal dominant polycystic kidney disease patients with enlarged kidney and liver. Yher Aper Dial 2006;10(4):333-41.

52. Singh S, Hariaran S. Renal replacement therapy in autosomal dominant polycystic kidney disease. Nephron 1991;57: 40-4.

53. Nakatani T, Kishimoto T, Tsujino T, et al. Urolocigal procedures for progressive renal dysfunction due to polycystic kidney disease. Hinyokika Kiyo 2000;46(9):645-50.
54. Segal AJ, Spataro RF, Barbaric ZL. Adult polycystic kidney disease: a review of 100 cases. J Urol 1977:121:711-3.

55. Gregoire JR, Torres VE, Holley KE. Renal epithelial hyperplastic and neoplastic proliferation in autosomal dominant polycystic kidney disease. Am J Kidney Dis 1987:9:27-34.

56. Vijay A, Vijay A, Pankaj P. Autosomal Dominant Polycistic Kidney disease: a comprehensive review. Int J Nephrol Urol 2010;2(1):172-92.

57. Lue YB, Anderson EE, Harrison HT. The surgical management of polycystic renal disease. Gynecol Obstet 1966;122:45-7.

58. Kumar S, Fan SL, Raftery MJ, Yaqoob MM. Long term outcome of patients with autosomal dominant polycystic kidney diseases receiving peritoneal dialysis. Kidney Int 2008;74:946-51.

59. Brunelli SM, Blanchette CM, Claxton AJ, Roy D, Rossetti $\mathrm{S}$, Gutierrez B. End-stage renal disease in autosomal dominant polycystic kidney disease: a comparison of dialysis-related utilization and costs with other chronic kidney diseases. Clinicoecon and Outcomes Res 2015;7:65-72.

60. Bretagnol A, Buchler $M$, Boutin JM, Nivet $H$, Lebranchu $Y$, Chauveau D. Renal transplantation in patients with autosomal dominant polycystic kidney disease: pre-transplantation evaluation and follow-uo. Nephrol Ther 2007;3(7):449-55.

61. Jacquet $A$, Pallet $N$, Le Kessler $M$, et al. Outcomes of renal transplantation in patients with autosomal dominant polycystic kidney disease: a nationwide longitudinal study. Transpl Int 2011;24:582-7.

62. Mehrabi, H. Fonouni, E. Ayoub, et al. A single center experience of combined liver kidney transplantation. Clin Transplant 2009;23(Suppl. 21):102-14.

63. Stiasny B, Ziebell D, Graf S, Hauser IA, Schulze BD. Clinical aspects of renal transplantation in polycystic kidney disease. Clin Nephrol 2002;58(1):16-24.

64. Hadimeri H, Norden G, Friman S, Nyberg G. Autosomal dominant polycystic kidney disease in a kidney transplant population. Nephrol Dial Transplant 1997;12:1431-6.

65. De Mattos AM, Olyaei AJ, Prather JC, Golconda MS, Barry JM, Norman DJ. Autosomal-dominant polycystic kidney disease as a risk factor for diabetes mellitus following renal transplantation. Kidney Int 2005;67(2):714-20.

66. Kanaan N, Devuyst O, Pirson Y. Renal transplantation in autosomal dominant polycystic kidney disease. Nat Rev Nephrol 2014;10:455-65.

67. Mosconi G, Persici E, Cuna V, et al. Renal transplant in patients with polycystic disease: the italian experience. Transplant Proc 2013;45(7):2635-40.

68. Kornasiewicz O, Dudek K, Bugajski M, Najnigier B, Krawczyk M. Choice of transplantation techniques and indications for liver transplantation in polycystic liver disease in patients with no signs of end-stage liver disease. Transplant Proc 2008;40(5):1536-8.

69. Ueno T, Barri YM, Netto GJ, et al. Liver and kidney transplantation for polycystic liver and kidney-renal function and outcome. Transplantation 2006;82(4):501-7.

70. Opelz G, Margreiter R, Döhler B. Prolongation of long-term kidney graft survival by a simultaneous liver transplant: the liver does it, and the heart does it too. Transplantation 2002;74(10):1390-4.

71. Thompson JA, Lake JR. The impact of MELD allocation on simultaneous liver-kidney transplantation. Curr Gastroenterol Rep 2009;11(1):76-82.

72. Rayner BL, Cassidy MJ, Jacobsen JE, Pascoe MD, Pontin AR, van Zyl Smit R. Is preliminary binephrectomy necessari in paqtients with autosomal dominant polycystic kidney disease undergoing renal transplantation? Clin Nephrol 1991;36(2):105-6.

73. Glassman DT, Nipkow L, Bartlett ST, Jacobs SC. Bilateral 
nephrectomy with concomitant renal graft transplantation for autosomal dominant policystic kidney disease. J Urol 2000; 164(3):661-4.

74. Tabibi A, Simforoosh N, Abadpour P, Gholamrezaie HR, Nafar M. Concomitant Nephrectomy of Massively Enlarged Kidneys and Renal Transplantation in Autosomal Dominant Polycystic Kidney Disease. Transpl Proc 2005;37(7):2939-40.

75. Fuller TF, Brennan TV, Feng S, Kang SM, Stock PG, Freise CE. End stage polycystic kidney disease: indications and timing of native nephrectomy relative to kidney transplantation. J Urol 2005;174(6):2284-8.

76. Wagner MD, Prather JC, Barry JM. Selective, concurrent bilateral nephrectomies at renal transplantation for autosomal dominant policystic kidney disease. J Urol 2007;177(6):2250-4.

77. Kramer A, Sausville J, Haririan A, Bartlett S, Cooper M, Phelan M. Simultaneous bilateral native nephrectomy and living donor renal transplantation are successful for polycystic kidney disease: the University of Maryland experience. J Urol. 2009;181(2):724-8.

78. Whitten MG, Van der Werf W, Belnap L. A novel approach to bilateral hand-assisted laparoscopic nephrectomy for autosomal dominant polycystic kidney disease. Surg Endosc 2006;20:679-84.

79. Nunes $P$, Mota A, Alves $R$, et al. Simultaneous Renal Transplantation and Native Nephrectomy in Patients With Autosomal-Dominant Polycystic Kidney Disease. Transpl Proc 2007;39(8):2483-5.

80. Sulikowski T, Tejchman K, Zietek Z, et al. Experience with autosomal dominant polycystic kidney disease in patients before and after renal transplantation: a 7-year observation. Transplant Proc 2009;41(1):177-80.

81. Lucas SM, Mofunanya TC, Goggins WC, Sundaram CP. Staged nephrectomy versus bilateral laparoscopic nephrectomy in patients with Autosomal Dominant Policystic Kidney. J Urol 2010;184(5):2054-9.

82. Neeff HP, Pisarski P, Tittelbach-Helmrich D, et al. One hundred consecutive kidney transplantations with simultaneous ipsilateral nephrectomy in patients with autosomal dominant polycystic kidney disease. NDT 2012;28(2):466-71.

83. Mak DK-C, llie CP, Abedin A, Gommersall L, Luscombe C, Golash A. Bilateral hand-assisted laparoscopic nephrectomy in adult polycystic kidney disease patients: a UK centre experience. J Med Life 2012;5(2):226-31.

84. Lee DI, Clayman RV. Hand-assisted laparoscopic nephrectomy in autosomal polycystic kidney disease. J Endurol 2004;18(4):379-82.

85. Lipke MC, Bargman V, Milgrom M, Sundaram CP. Limitation s of laparoscopi for bilateral nephrectomy for autosomal dominant polycystic kidney disease. J Urol 2007;177(2):627-31.

86. Shumate AM, Bahler CD, Goggins WC, Sharfuddin AA,
Sundaram CP. Native Nephrectomy with Renal Transplantation is Associated with a Decrease in Hypertension Medication Requirements in Autosomal Dominant Polycystic Kidney Disease. J Urol 2016;195(1):141-6.

87. Kirkman MA, Van Dellen D, Babatunde SM, et al. Native nephrectomy for autosomal dominant polycystic kidney disease: before or after kidney transplantation? BJU Int 2010; 108:590-4.

88. Skauby $\mathrm{MH}, \varnothing$ yen $\mathrm{O}$, Hartman A, Leivestad T, Wadström J. Kidney Transplantation With and Without Simultaneous Bilateral Native Nephrectomy in Patients With Polycystic Kidney Disease: A Comparative Retrospective Study. Transplantation 2012;94(4):383-8.

89. Desai MR, Nandkishore SK, Ganpule A, Thimmegowda M. Pretransplant laparoscopic nephrectomy in adult polycystic kidney disease: a single centre experience. BJU Int 2008; 101(1):94-7.

90. Verhoest G, Delreux A, Mathieu R, et al. Transperitoneal laparoscopic nephrectomy for autosomal dominant polycystic kidney disease. JSLS 2012;16:437-42.

91. Williasmson A, Paterson S, Erolin C, Sweeney C, Townell N, Nabi G. Laparoscopic nephrectomy for adult polycystic kidney disease: safety, feasibility and early outcomes. J Endourol 2014;28(11);1268-77.

92. Ahmad SB, Inoute B, Phelan MS, et al. Live donor renal transplant with simultaneous bilateral nephrectomy for autosomal dominant polycystic kidney disease is feasible and satisfactory at long-term follow-up. Transplantation 2016;100(2):407-15.

93. Knispel HH, Klan R, Offermann G, Miller K. Transplantation in autosomal dominant polycystic kidney disease without nephrectomy. Urol Int 1996: 56(2):75-8.

94. Muller TF, Brennan TV, Feng S, Kang SM, Stock PG, Freise CE. End Stage polycystic kidney disease: indication and timing of native nephrectomy relative to kidney transplantation. J Urol 2005;174(6):2284-8.

95. Rozanski J, Kozlowska I, Myslak M, et al. Pretransplant nephrectomy in patients with autosomal dominant polycystic kidney disease. Transpl Proc 2005;37(2):666-8.

96. Cohen D, Timist MO, Chrétien T, Thiounn N, Vassilu V, Mamzer MF. Place of nephrectomy in patients with autosomal dominant polycystic kidney disease waiting for renal transplantation. Progr Urol 2008;18(10):642-9.

97. Patel P, Horsefield C, Compton F, Taylor J, Koffman G, Olsburg J. Native nephrectomy in transplant patients with autosomal dominant polycystic kidney disease. Ann R Coll Surg Engl 2011;93:391-5.

98. Lasaponara F. PKD open nephrectomy: minimally invasive tecnique. Giornale di tecniche Nerologiche e Dialitiche 2014;26(2):209-15. 